\title{
Design of Environmental Monitoring Device on Transmission Line
}

\author{
Haokun Guo ${ }^{1, a}$ \\ ${ }^{1}$ Department of Electronic Information Engineering, Jiangyin Polytechnic College, Jiangyin Jiangsu \\ 214405, China \\ aghk1222@126.com
}

Keywords: Transmission line; Monitoring device; Monitor; Transmission; Environmental data

\begin{abstract}
In recent years, various types of high-voltage transmission lines on the frequent accidents. Some of these serious accidents can even lead to a large area of power outages. A large part of these accidents is caused by bad dangers. Therefore, it is necessary to design the environmental monitoring device on the high voltage transmission line. This paper designs an environment meteorological monitoring device for overhead transmission lines. The device can monitor the environmental data on the transmission line in real time, such as wind speed, wind direction, air temperature, humidity, rainfall and light radiation. The device was installed in a transmission line. The environmental data of December 2015 is sorted out, and the data is small compared to the actual data. This indicates that the device is functioning correctly.
\end{abstract}

\section{Introduction}

The global ecological environment and the rapid depletion of fossil fuels and other issues become increasingly serious. The construction of smart grid has become a new direction for the development of today's world power grid [1]. In recent years, various types of high-voltage transmission lines on the frequent accidents. Some of these serious accidents can even lead to a large area of power outages. A large part of these accidents is caused by bad dangers. Therefore, it is necessary to design the environmental monitoring device on the high voltage transmission line $[2,3]$.

At present, the monitoring device on the transmission line was studied by some scholars $[4,5,6]$. In this paper, an aerial transmission line environmental meteorological monitoring device is designed. The device can achieve centralized monitoring and reliable transmission of parameters such as wind speed, wind direction, temperature, humidity, rainfall and light radiation. The device can meet the requirements of China State Grid Corporation, such as accuracy, reliability and stability.

\section{Overall Design}

These overhead transmission line environmental monitoring devices are installed on the tower of the overhead transmission lines that need to be monitored. As shown in Fig. 1, a monitoring terminal is installed on each of the transmission line towers that need to be monitored. Select one of the several terminals as a concentrator. The monitoring terminal transmits the collected meteorological information to the concentrator through the digital broadcasting station, and then the concentrator transmits it to the background computer through the digital transmission station. Expert analysis software is installed in the background on the machine. The software which can provide a variety of weather data for dispatchers contains functions such as displaying data, storing data, and so on. 


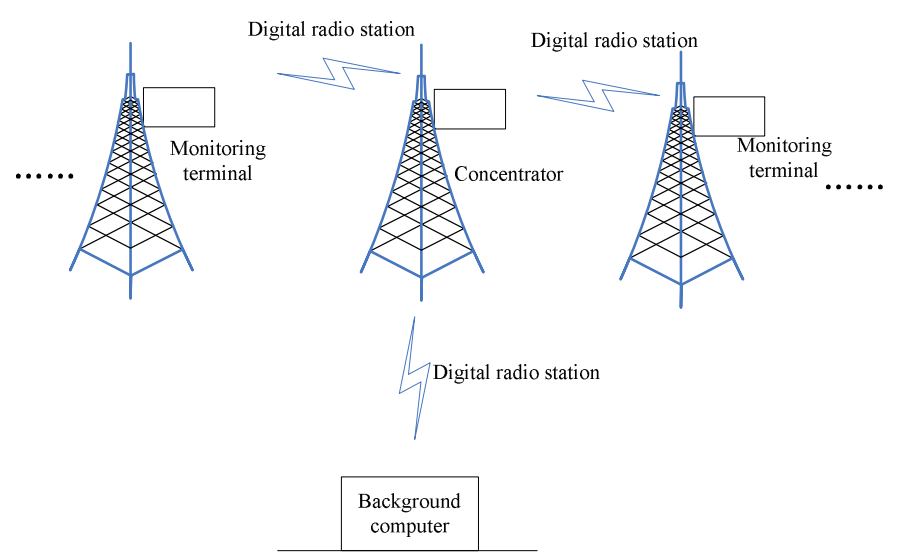

Figure 1. Finite Simplified schematic of the monitoring device

\section{Key Module Design}

Monitor Terminal Design. Monitoring terminal is composed of STM32 microcontroller, GPS module, sensor module, power supply module, digital radio station, as shown in Fig. 2. Among them, the sensor module includes ambient temperature and humidity sensor (SHT71), wind speed sensor (BCQ-FS-BA), sunshine radiation sensor (GZD system) and rainfall sensor (WD211). The sensor stores the measured environmental parameters in the STM32 microcontroller and sends the data to the concentrator via a digital radio station. During the operation of the unit, the power supply module supplies power to the monitoring terminal. And the GPS module acts as proof of time, so as to ensure the synchronization of data upload.

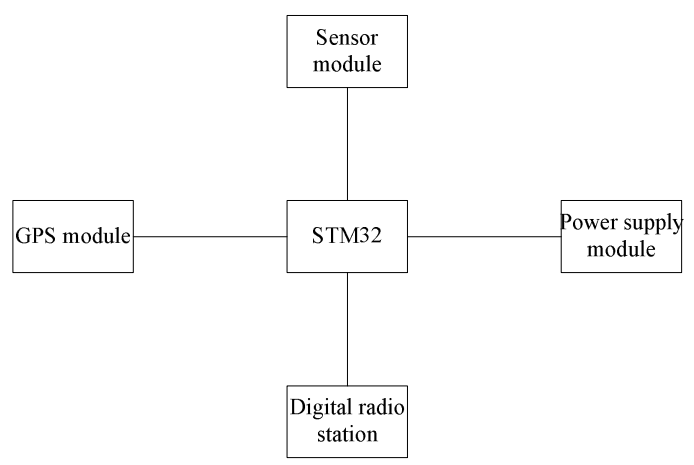

Figure 2. Finite Structure diagram of the monitoring terminal

Concentrator Design. Concentrator is composed of STM32 microcontroller, GPS module, sensor module, power supply module, digital radio station, EUDO module, as shown in Fig.3. The sensor module includes ambient temperature and humidity sensor (SHT71), wind speed sensor (BCQ-FS-BA), sunshine radiation sensor (GZD system) and rain sensor (WD211). It can receive the monitoring terminal measured by the environmental meteorological parameters and sensors measured by the environmental meteorological parameters stored in the STM32 microcontroller, through the digital radio station to send these data to the background computer. If the distance between the concentrator and the background PC is more than $40 \mathrm{~km}$, it is transmitted through the EUDO module. 


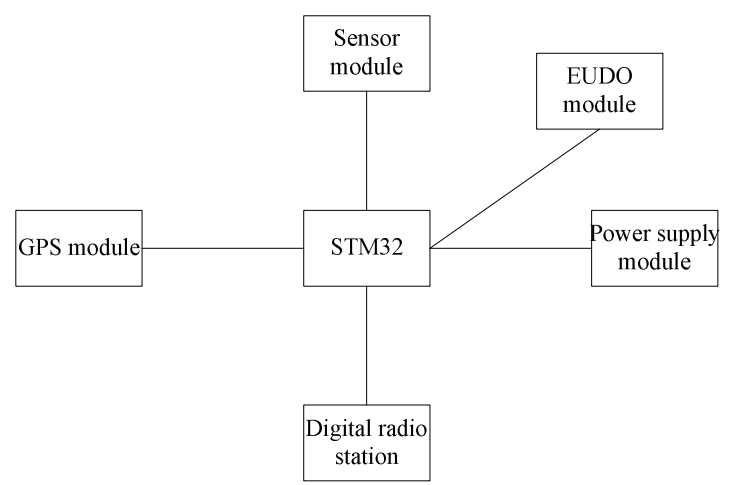

Figure 3. Finite Structure diagram of the concentration

Power Supply Module Design. Power supply module is an intelligent protection of the induction power supply device [7], as shown in Figure 4. The core was crossed by a bus. In the normal operation of the grid, a bus will have the very constant amplitude of the AC current, so the line will produce alternating magnetic field around the core to produce alternating magnetic flux. The flux will induce alternating current in the induction coil. The induced current is shown as an electromotive force output on the sampling resistor. The induced electromotive force is rectified by the rectifier circuit and becomes the pulsating DC voltage. Pulsating DC voltage through the transformer bleeder circuit will be pulsating DC voltage spike energy is discharged to the resistance discharge circuit. When the transformer bleeder circuit over voltage occurs, the excess power through the resistor bleed circuit is released. The ripple of the output voltage of the rectifier circuit and the transformer bleeder circuit is filtered out by filtering the capacitor. The STM8 microcontroller control system is used to monitor the output voltage of the rectifier circuit and the output voltage of the transformer discharge circuit and to control the switching between the output voltage of the rectifier circuit and the output voltage of the transformer bleeder circuit. Finally, the voltage is reduced by the regulated voltage to the operating voltage supply load.

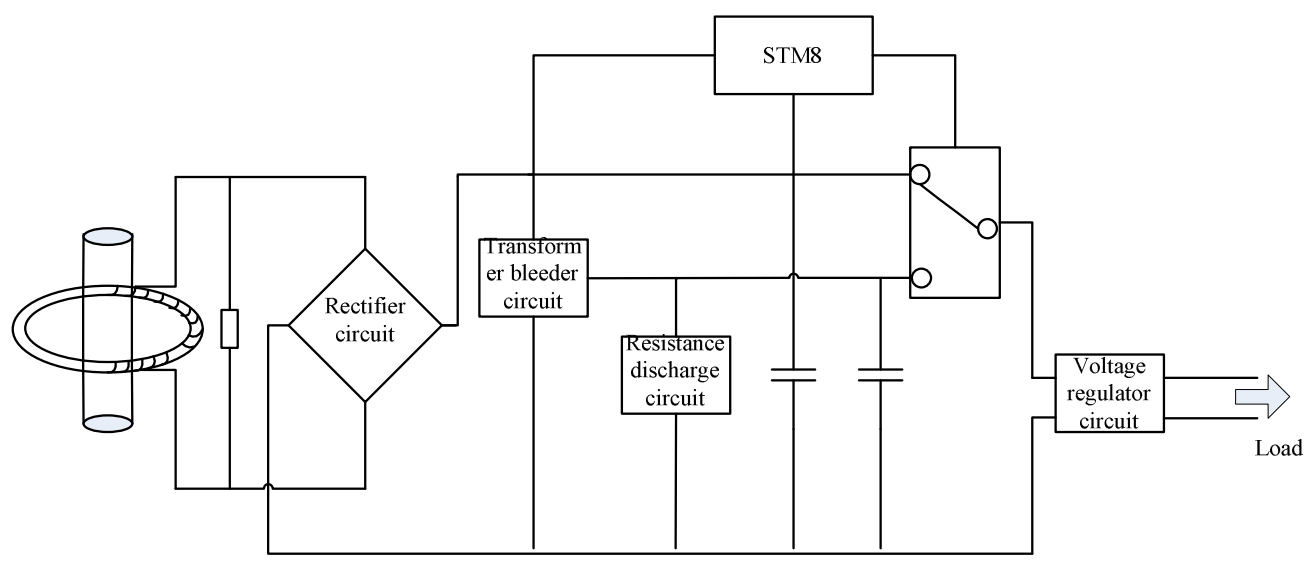

Figure 4. Finite Structure diagram of the power modules

\section{Measured Data}

According to the above design of the device, the monitoring terminal was designed. The device is installed on a $110 \mathrm{kV}$ transmission line, the background of the host computer is installed in the local power supply company. Using the device to measure a live line of the transmission line data shown in Fig. 5, these data include temperature, humidity, atmospheric pressure and wind speed. The design of 
the transmission line on the environmental weather monitoring device in a long time can work properly, no obvious failure. Power supply is normal, no power outages. The measured environmental data is consistent with the actual situation.

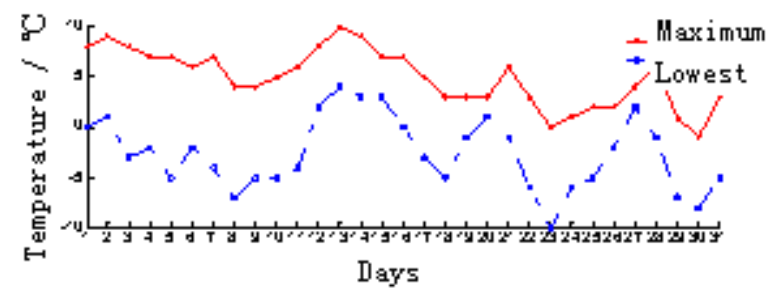

(a) Temperature data of December 2015

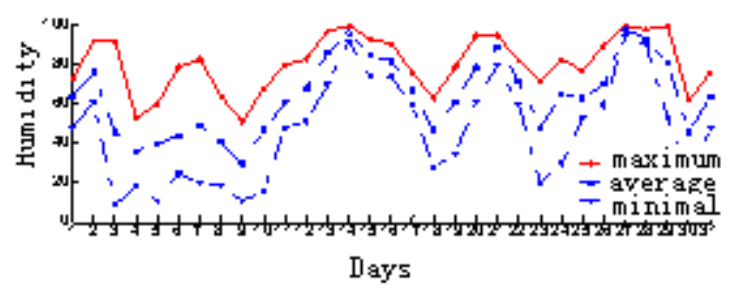

(b) Humidity data of December 2015

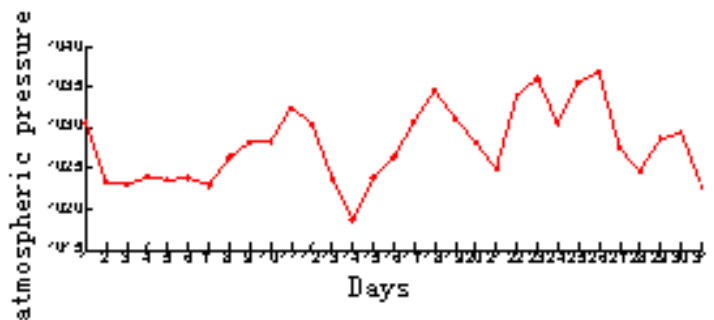

(c) Atmospheric pressure data of December 2015

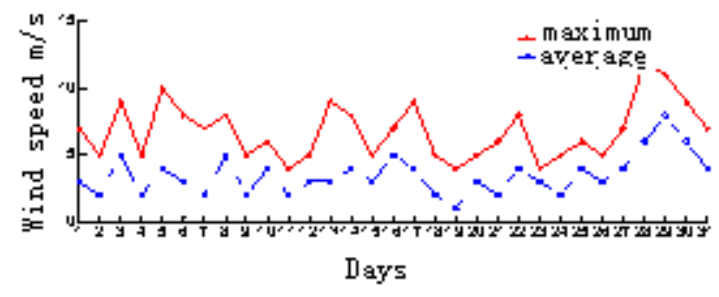

(d) Wind speed data of December 2015

Figure 5. Finite Field environmental data

\section{Summary}

An aerial transmission line environmental meteorological monitoring device was designed. The device consists of a monitoring terminal, a concentrator, and a background PC. The device can achieve centralized monitoring and reliable transmission of parameters such as wind speed, wind direction, temperature, humidity, rainfall and light radiation. The device can monitor the important meteorological parameters of important transmission lines and disaster-prone areas, so as to provide decision information for production operation management and dispatch. This paper verifies the reliability, effectiveness and practicability of the device by actually measuring the environmental data of a transmission line.

\section{Acknowledgements}

Research Subject of Jiangyin Polytechnic College of China. 


\section{References}

[1] GUO H K, WU J J. Application of Agent Technology in China's Smart Grid Construction. Power System and Clean Energy. Vol.30 (2014) No. 13, p. 12-16.

[2] LI Q F, FAN Z, WU Q, et al. Investigation of Ice-Covered Transmission Lines and Analysis on Transmission Line Failures Caused by Ice-Coating in China. Power System Technology. Vol.32 (2008) No. 9, p. 33-36.

[3] WANG S H. Analysis of Typical Ice Accidents of Transmission Lines and Preventing Techniques. High Voltage Apparatus. Vol.46 (2010) No. 10, p. 85-89.

[4] CHENG H L, SUN S. A Photovoltaic and Super- Capacitor Based Power Supply for Transmission Line Monitoring Equipment. Electrical Measurement \& Instrumentation. Vol.51 (2014) No. 9 p. 97-101.

[5] GUO H K, HENG S K, YING Z F, et al. Dynamic Capacity-increase Online Monitoring Device of Transmission Lines. Journal of Electric Power. Vol.27 (2012) No. 5, p. 459-462.

[6] GUO H K, HENG S K, YING Z F, et al. Research on On-line Monitoring System for Overhead Transmission Line Conductors Galloping. Science Technology and Engineering. Vol.12 (2012) No. 14, p. 184-186, 194.

[7] GUO H K, WU J J. A New Equipment of Rechargeable Induction Power Supply at High Voltage. Electrical Measurement \& Instrumentation. Vol.51 (2014) No. 3, p. 86-90. 